\title{
The strong spectral extension property does not imply the multiplicative Hahn-Banach property
}

by

\section{A. SoŁtysiak (Poznań)}

\begin{abstract}
An example is given of a semisimple commutative Banach algebra that has the strong spectral extension property but fails the multiplicative Hahn-Banach property. This answers a question posed by M. J. Meyer in [4].
\end{abstract}

Let $A$ be a commutative Banach algebra. For an element $a \in A$ we denote by $r_{A}(a)$ and $\sigma_{A}(a)$ its spectral radius and spectrum in $A$ respectively. An extension of $A$ is a Banach algebra $B$ such that $A$ is algebraically embedded in $B$. Hence we can view $A$ as a subalgebra (not necessarily closed) of $B$. If $B$ is an extension of $A$, then $\sigma_{B}(a) \cup\{0\} \subset \sigma_{A}(a) \cup\{0\}$ and therefore $r_{B}(a) \leq r_{A}(a)$ for all $a \in A$. In [4] M. J. Meyer investigated the following three conditions:

(1) $r_{B}(a)=r_{A}(a)$ for all $a \in A$ and all extensions $B$ of $A$;

(2) $\sigma_{B}(a) \cup\{0\}=\sigma_{A}(a) \cup\{0\}$ for all $a \in A$ and all extensions $B$ of $A$;

(3) every multiplicative linear functional on $A$ has a (multiplicative linear) extension to every commutative extension $B$ of $A$.

A Banach algebra $A$ has the spectral extension property if it satisfies (1), the strong spectral extension property if it satisfies (2), and the multiplicative Hahn-Banach property if it satisfies (3).

Notice that if $A$ is commutative we can restrict ourselves to commutative extensions when dealing with properties (1) and (2) (cf. [5, Thm. 11.22]). Obviously, if a commutative Banach algebra has the multiplicative HahnBanach property, then it has the strong spectral extension property, and this in turn implies the spectral extension property. M. J. Meyer in [4, Ex. 2] gave an example of a semisimple commutative Banach algebra with the spectral extension property which does not have the strong spectral extension property. He asked if for a commutative semisimple Banach algebra the strong $46 \mathrm{~A} 22$

2000 Mathematics Subject Classification: Primary 46J05; Secondary 46J20, 46J30, 
spectral extension property implies the multiplicative Hahn-Banach property. We show by an example that the answer to this question is negative.

For an element $a$ of a Banach algebra $A$ the permanent radius $r_{\mathrm{p}}(a)$ is defined to be the infimum of $r_{B}(a)$, where $B$ runs through all extensions of the algebra $A$. This notion plays an important role in characterization of the spectral extension property of commutative semisimple Banach algebras. For convenience of the reader we state the following three results of Meyer which are crucial for our reasoning.

(A) $[4$, Prop. 2(1)] Let $A$ be a Banach algebra and let $a \in A$. If $a x=x$ or $x a=x$ for some nonzero $x \in A$, then $r_{\mathrm{p}}(a) \geq 1$.

(B) $[4$, Thm. 1] A semisimple commutative Banach algebra $A$ has the spectral extension property if and only if for every closed subset $F$ of the maximal ideal space $\mathfrak{M}(A)$ that does not contain the Shilov boundary $\Gamma(A)$ of $A$, there exists an element $a \in A$ such that its Gelfand transform $\widehat{a}$ is zero on $F$ and $r_{\mathrm{p}}(a)>0$.

(C) $[4$, Thm. 2] A semisimple commutative Banach algebra $A$ has the strong spectral extension property if and only if it has the spectral extension property and $\widehat{a}(\Gamma(A)) \cup\{0\}=\widehat{a}(\mathfrak{M}(A)) \cup\{0\}$ for every $a \in A$.

ExAmple. Take the open ball $B(0,1)=\left\{(z, w):|z|^{2}+|w|^{2}<1\right\}$ in $\mathbb{C}^{2}$ and closed ball $\bar{B}(0,2)=\left\{(z, w):|z|^{2}+|w|^{2} \leq 4\right\}$. Let $\mathcal{A}$ be the algebra of all continuous functions on $\bar{B}(0,2)$ that are holomorphic on $B(0,1)$. Then $\mathcal{A}$ is a semisimple commutative Banach algebra. The maximal ideal space of $\mathcal{A}, \mathfrak{M}(\mathcal{A})$, can be identified with the ball $\bar{B}(0,2)$ in such a way that the Gelfand transforms $\widehat{f}$ coincide with the functions $f$. The Shilov boundary $\Gamma(\mathcal{A})$ is then the set $\left\{(z, w): 1 \leq|z|^{2}+|w|^{2} \leq 4\right\}$. Moreover if $F$ is a closed subset of $\bar{B}(0,2)$ not containing the Shilov boundary, then there is an open subset $U$ of $\left\{(z, w): 1 \leq|z|^{2}+|w|^{2} \leq 4\right\}$ with closure disjoint from $F$. The algebra $\mathcal{A}$ contains a function $f$ which is zero on $F$ and 1 on $U$. Also $\mathcal{A}$ contains a nonzero function $g$ with support in $U$. Then $f g=g$ and by $(\mathrm{A})$ we have $r_{\mathrm{p}}(f) \geq 1$. By (B) the algebra $\mathcal{A}$ has the spectral extension property. From the well-known property of holomorphic functions of several variables (see e.g. [3, Thm. 1.2.6]) it follows that if a function $f$ from $\mathcal{A}$ takes value $c$ at a point $(z, w) \in B(0,1)$, then there exists a point $\left(z_{0}, w_{0}\right)$ such that $\left|z_{0}\right|^{2}+\left|w_{0}\right|^{2}=1$ and $f\left(z_{0}, w_{0}\right)=c$. Therefore $\sigma_{\mathcal{A}}(f)=f(\bar{B}(0,2))=f\left(\left\{(z, w): 1 \leq|z|^{2}+|w|^{2} \leq 4\right\}\right)$. By (C) the algebra $\mathcal{A}$ has the strong spectral extension property. But obviously it does not have the multiplicative Hahn-Banach property since the evaluation functionals at the points of the unit ball $B(0,1)$ do not extend to the Banach algebra of continuous functions on $\Gamma(\mathcal{A})=\left\{(z, w): 1 \leq|z|^{2}+|w|^{2} \leq 4\right\}$. 
In the remaining part of the paper we shall try to clarify the difference between conditions (2) and (3). First notice that we can restrict ourselves to unital Banach algebras and unital embeddings. Indeed, if $j: A \rightarrow B$ is an embedding and $A_{1}$ and $B_{1}$ are unitizations of $A$ and $B$ respectively (regardless of whether $A$ or $B$ have units), then we extend $j$ to $j_{1}: A_{1} \rightarrow B_{1}$ by $j_{1}\left(a+\lambda 1_{A}\right)=j(a)+\lambda 1_{B}$, where $1_{A}$ and $1_{B}$ denote units adjoined to $A$ and $B$ respectively. Then $\sigma_{A_{1}}(a)=\sigma_{A}(a) \cup\{0\}$ and $\sigma_{B_{1}}(a)=\sigma_{B}(a) \cup\{0\}$ (see [1]). Hence condition (2) may be written in the form

$$
\sigma_{A_{1}}(a)=\sigma_{B_{1}}(a)
$$

Moreover every multiplicative linear functional $\varphi$ on $A$ extends to a multiplicative linear functional $\varphi_{1}$ on $A_{1}$ in the obvious way. Hence if $\varphi$ extends to $B$ then $\varphi_{1}$ extends to $B_{1}$. Therefore we shall assume in what follows that all algebras are unital and if $B$ is an extension of an algebra $A$, then $A$ is a subalgebra of $B$ and the unit of $B$ belongs to $A$. Moreover we shall denote the common unit of $A$ and $B$ by 1 .

For a Banach algebra $A$ we denote by $I_{A}\left(a_{1}, \ldots, a_{n}\right)$ the (two-sided) ideal in $A$ generated by the elements $a_{1}, \ldots, a_{n} \in A$. The Harte spectrum $\sigma_{A}\left(a_{1}, \ldots, a_{n}\right)$ is the set of all $n$-tuples $\left(\lambda_{1}, \ldots, \lambda_{n}\right) \in \mathbb{C}^{n}$ such that either the left or the right ideal in $A$ generated by the elements $a_{1}-\lambda_{1}, \ldots, a_{n}-\lambda_{n}$ is proper. (Here we write $a_{j}-\lambda_{j}$ instead of $a_{j}-\lambda_{j} 1$.) For a single element $a \in A$ the Harte spectrum $\sigma_{A}(a)$ coincides with the usual spectrum of $a$. It is well known that if the algebra $A$ is commutative, then $\sigma_{A}\left(a_{1}, \ldots, a_{n}\right)$ $=\left\{\left(\varphi\left(a_{1}\right), \ldots, \varphi\left(a_{n}\right)\right): \varphi\right.$ is a multiplicative linear functional on $\left.A\right\}$.

Now we deal with extensions of multiplicative linear functionals to superalgebras.

Proposition 1. Let $A$ be a commutative Banach algebra with unit and let $B$ be its unital and commutative extension. The following conditions are equivalent:

(i) For every multiplicative linear functional $\varphi$ on $A$ there exists a multiplicative linear functional $\psi$ on $B$ such that $\left.\psi\right|_{A}=\varphi$;

(ii) $\sigma_{A}\left(a_{1}, \ldots, a_{n}\right) \subset \sigma_{B}\left(a_{1}, \ldots, a_{n}\right)$ for all finite subsets $\left\{a_{1}, \ldots, a_{n}\right\}$ of $A$;

(iii) if $I_{A}\left(a_{1}, \ldots, a_{n}\right) \neq A$, then $I_{B}\left(a_{1}, \ldots, a_{n}\right) \neq B$, for all finite subsets $\left\{a_{1}, \ldots, a_{n}\right\}$ of $A$

(iv) every proper ideal of $A$ is contained in a proper ideal of $B$;

(v) every maximal ideal of $A$ is contained in a maximal ideal of $B$.

The proof is straightforward. We leave it as an exercise to the interested reader. 
Remarks. 1. Commutativity of $B$ is essential only in the proof of the implication $(\mathrm{v}) \Rightarrow(\mathrm{i})$. Clearly this implication need not be true if $B$ is noncommutative. To see this we take a compact operator $T$ on a separable infinite-dimensional Hilbert space $\mathcal{H}$. Let $A$ be the closed subalgebra of $B(\mathcal{H})$ (the algebra of all bounded linear operators on $\mathcal{H}$ ) generated by $T$ and the identity, and let $B=B(\mathcal{H})$. Then every maximal ideal of $A$ is contained in the ideal of compact operators which is maximal in $B(\mathcal{H})$, but clearly no multiplicative linear functional on $A$ extends to $B(\mathcal{H})$.

2. The equivalence (i) $\Leftrightarrow($ ii) was also proved by A. Kokk [2, Cor. 4].

To formulate the next result we introduce the following notation. For a Banach algebra $B$ let $N_{B}$ denote its set of noninvertible elements. If $B$ is an extension of the algebra $A$ and $a_{1}, \ldots, a_{n} \in A$, then

$$
\tau_{B}\left(a_{1}, \ldots, a_{n}\right)=\left\{\left(\lambda_{1}, \ldots, \lambda_{n}\right) \in \mathbb{C}^{n}: I_{A}\left(a_{1}-\lambda_{1}, \ldots, a_{n}-\lambda_{n}\right) \subset N_{B}\right\} .
$$

This notion was introduced by A. Wawrzyńczyk in [6]. He showed, among other things, that $\tau_{B}$ is a subspectrum in the sense of Żelazko (see [7]) on a commutative Banach algebra $A$.

Proposition 2. Let $A$ be a commutative Banach algebra with unit and let $B$ be its unital extension. The following conditions are equivalent:

(i) $\sigma_{A}(a)=\sigma_{B}(a)$ for every $a \in A$;

(ii) $\sigma_{A}\left(a_{1}, \ldots, a_{n}\right) \subset \tau_{B}\left(a_{1}, \ldots, a_{n}\right)$ for all finite subsets $\left\{a_{1}, \ldots, a_{n}\right\}$ of $A$;

(iii) if $I_{A}\left(a_{1}, \ldots, a_{n}\right) \neq A$, then $I_{A}\left(a_{1}, \ldots, a_{n}\right) \subset N_{B}$, for all finite subsets $\left\{a_{1}, \ldots, a_{n}\right\}$ of $A$;

(iv) for every proper ideal $I$ of $A, I \subset N_{B}$;

(v) for every maximal ideal $M$ of $A, M \subset N_{B}$;

(vi) the algebra $A$ is inverse closed in $B$, i.e. for every $a \in A$, if $a^{-1} \in B$, then $a^{-1} \in A$.

We leave the easy proof as an exercise.

Remark. Notice that in Proposition 2 we do not assume that the algebra $B$ is commutative.

\section{References}

[1] A. Ya. Helemskii, Banach Algebras and Locally Convex Algebras, Oxford Univ. Press, Oxford, 1993.

[2] A. Kokk, Spectral systems with the one-way spectral mapping property, Acta Comment. Univ. Tartuensis Math. 5 (2001), 65-73.

[3] S. G. Krantz, Function Theory of Several Complex Variables, Wiley, New York, 1982.

[4] M. J. Meyer, The spectral extension property and extension of multiplicative linear functionals, Proc. Amer. Math. Soc. 112 (1991), 855-861. 
[5] W. Rudin, Functional Analysis, McGraw-Hill, New York, 1991.

[6] A. Wawrzyńczyk, On subspectra generated in subalgebras, Bull. London Math. Soc., to appear.

[7] W. Żelazko, An axiomatic approach to joint spectra I, Studia Math. 64 (1979), 249261.

Faculty of Mathematics and Computer Science

Adam Mickiewicz University

Umultowska 87

61-614 Poznań, Poland

E-mail: asoltys@amu.edu.pl

Received February 28, 2002

Revised version May 16, 2002 